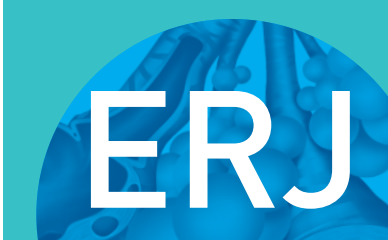

open research
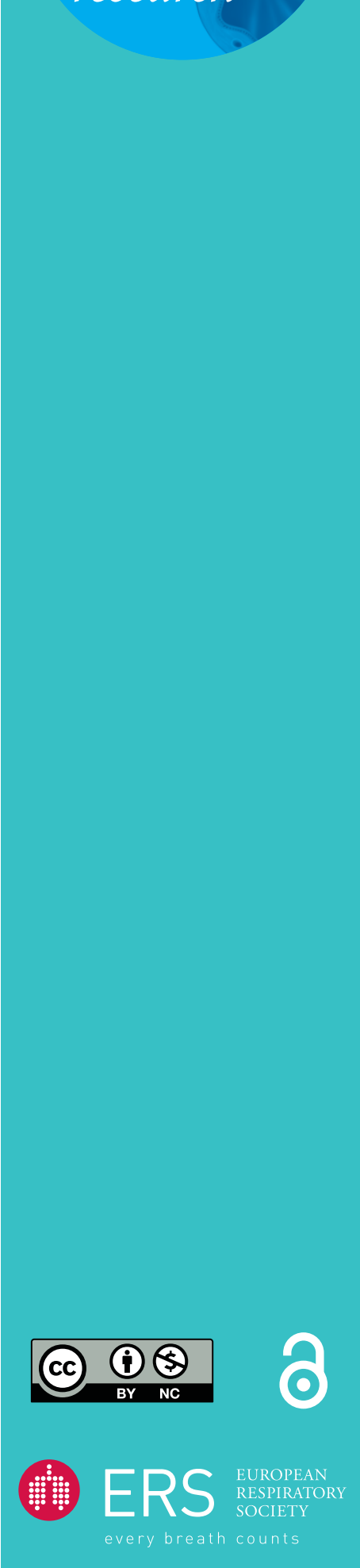

\title{
Increasing mortality rate due to rheumatoid arthritis-related lung diseases in Japan
}

\author{
To the Editor:
}

The quality of life of rheumatoid arthritis (RA) patients has dramatically improved in the approximately two decades since biological agents were first introduced for its treatment [1]. However, whether current treatment strategies for RA have helped improve the prognosis of patients suffering from complications due to RA remains controversial. National data from the USA have shown that the mortality rate of RA-associated interstitial lung disease (ILD), a major complication associated with a poor prognosis in RA patients, seems to be gradually increasing, whereas the mortality rate of patients with any type of RA-associated disease has been decreasing [2].

Japanese people have been found to be more susceptible to ILD than people of other ethnic backgrounds. In fact, drug-induced interstitial pneumonia occurs in Japanese people significantly more frequently than people of other ethnicities (e.g. incidence of gefitinib-induced ILD in Japan and the USA: 3.98\% and 0.3\%, respectively), and acute exacerbation of idiopathic pulmonary fibrosis is seen more frequently among Japanese than those of other ethnicities [3,4]. However, the annual trends in RA-ILD-related death have not been compared before and after the introduction of biological agents for RA treatment among such ILD-susceptible patients in Japan.

Using records stored in the Vital Statistics database of the Ministry of Health, Labour and Welfare of Japan from 1998 to 2016, we captured all deaths from any RA-related cause, defined by the 10th revision of the International Classification of Diseases (ICD-10) codes M05.0-M05.9 and M06.0-M06.9 (except for M06.1 coding adult-onset Still's disease), and death from RA-associated lung disease (LD) using ICD-10 code M05.1, which encodes rheumatoid lung disease including any types of pulmonary manifestations of RA. The database does not distinguish RA-ILD from RA-LD, but it is known that RA-ILD accounts for most RA-LD cases [5]. Airway involvement, another common pulmonary manifestation of RA, is included under the code for RA-LD, but lung infection is not. Although we were unable to calculate the age-adjusted rates due to a lack of data, the rates of death due to RA-ILD among deaths from any type of RA-related cause have been increasing in both men and women with statistical significance according to a trend test (figure 1).

These results are consistent with the report from the USA [2]. While the factors underlying these trends remain unclear, several have been hypothesised. First, the elderly population is increasing in Japan, which may influence the increasing rate of RA-LD mortality among deaths from any RA-related cause, probably because elderly people are more likely to develop ILD than younger people [6]. Second, the increased use of biological agents may be associated with an increasing rate of RA-ILD-related death. The timing of the increasing rates of RA-ILD-related death seems to coincide with the timing of the introduction of biological agents (from 2002) [1]. While death from ILD induced by biological agents is categorised as drug-induced pneumonitis (ICD-10 codes J70.2-J70.4), it is challenging to distinguish ILD induced by RA itself from that induced by drugs. Deaths from ILD induced by biological agents may be included among RA-LD (ICD-10 code M05.1) even if lung infection, such as Pneumocystis jirovecii pneumonia, has been excluded from the differential diagnosis. Third, the decreasing use of glucocorticoids might contribute to

@ERSpublications

The rates of death due to RA-LD among deaths from any type of RA-related cause have been increasing in Japan. A large-scale study is required to explain this trend. http://bit.ly/2Ew8fhW

Cite this article as: Yamasue $\mathrm{M}$, Komiya $\mathrm{K}$, Takahashi OIncreasing mortality rate due to rheumatoid arthritis-related lung diseases in Japan. ERJ Open Res 2020; 6: 00217-2019 [https:// doi.org/10.1183/23120541.00217-2019]. 

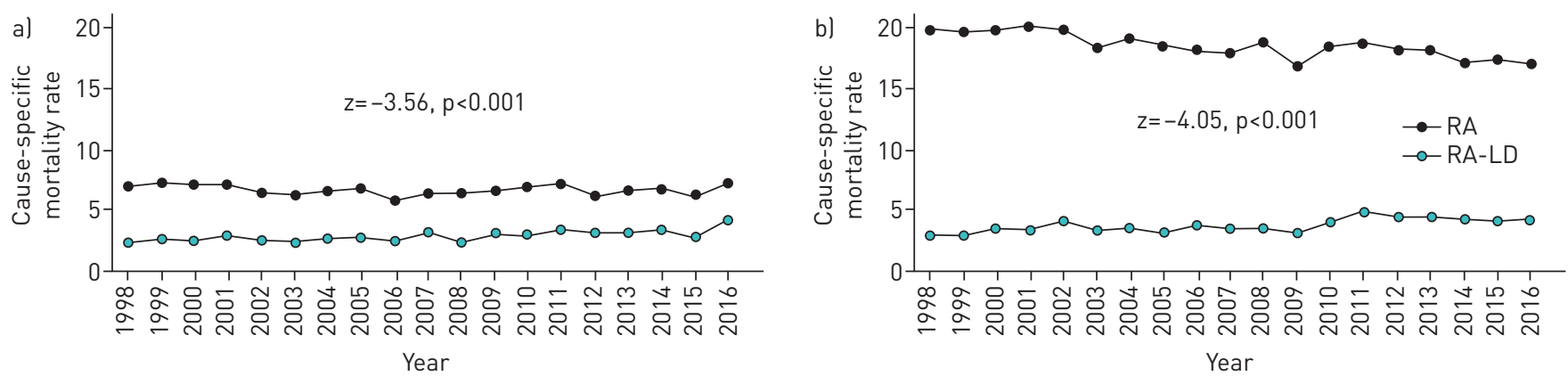

FIGURE 1 The annual trends in cause-specific death from rheumatoid arthritis (RA) and RA-associated lung disease (LD) among a) males and b) females in Japan.

the increasing number of deaths from RA-ILD. In fact, glucocorticoid use has been declining as biological agents have been increasingly frequently used for RA treatment [1]. Glucocorticoids may be effective for some types of RA-ILD, such as nonspecific interstitial pneumonia, despite evidence to this end being limited and only obtained from nonrandomised controlled trials $[7,8]$, so reducing their use might accelerate the development and progression of ILD among RA patients. However, glucocorticoids seem to have no significant beneficial effects on the usual interstitial pneumonia pattern, which is the major type of ILD in patients with RA [9]. Whether or not reducing glucocorticoid use contributes to an increase in the mortality rate of RA-ILD remains controversial. Finally, the growing recognition of RA-LD among physicians may increase the reporting of RA-LD-related mortality. A large-scale study is required to assess the causal relationship between these possible factors and the increasing rate of RA-LD-related death among overall RA-related death.

\section{Mari Yamasue $\odot^{1}$, Kosaku Komiya ${ }^{1}$, Osamu Takahashi ${ }^{2}$ and Jun-ichi Kadota ${ }^{1}$}

${ }^{1}$ Dept of Respiratory Medicine and Infectious Diseases, Oita University Faculty of Medicine, Yufu, Japan. ${ }^{2}$ Graduate School of Public Health, St Luke's International University, OMURA Susumu \& Mieko Memorial St Luke's Center for Clinical Academia, Tokyo, Japan.

Correspondence: Kosaku Komiya, Dept of Respiratory Medicine and Infectious Diseases, 1-1 Idaigaoka, Hasama-machi, Yufu, Oita 879-5593, Japan. E-mail: komiyakh1@oita-u.ac.jp

Received: 25 June 2019 | Accepted after revision: 12 Dec 2019

Conflict of interest: None declared.

\section{References}

1 Yamanaka H, Seto Y, Tanaka E, et al. Management of rheumatoid arthritis: the 2012 perspective. Mod Rheumatol 2013; 23: 1-7.

2 Olson AL, Swigris JJ, Sprunger DB, et al. Rheumatoid arthritis-interstitial lung disease-associated mortality. Am J Respir Crit Care Med 2011; 183: 372-378.

3 Azuma A, Kudoh S. High prevalence of drug-induced pneumonia in Japan. Japan Med Assoc J 2007; 50: 405-411.

4 Jo HE, Corte TJ. Nintedanib for idiopathic pulmonary fibrosis in the Japanese population. Respirology 2017; 22: 630-631.

5 Turesson C, O'Fallon WM, Crowson CS, et al. Extra-articular disease manifestations in rheumatoid arthritis: incidence trends and risk factors over 46 years. Ann Rheum Dis 2003; 62: 722-727.

6 Raghu G, Chen SY, Hou Q, et al. Incidence and prevalence of idiopathic pulmonary fibrosis in US adults 18-64 years old. Eur Respir J 2016; 48: 179-186.

7 Kim EJ, Collard HR, King TE Jr. Rheumatoid arthritis-associated interstitial lung disease: the relevance of histopathologic and radiographic pattern. Chest 2009; 136: 1397-1405.

8 Suda T. Up-to-date information on rheumatoid arthritis-associated interstitial lung disease. Clin Med Insights Circ Respir Pulm Med 2015; 9: Suppl. 1, 155-162.

9 Kim EJ, Elicker BM, Maldonado F, et al. Usual interstitial pneumonia in rheumatoid arthritis-associated interstitial lung disease. Eur Respir J 2010; 35: 1322-1328. 